\title{
SPECIMEN CHARGING ON THIN FILMS WITH ONE CONDUCTING LAYER: DISCUSSION OF PHYSICAL PRINCIPLES
}

Robert M. Glaeser ${ }^{1,2}$ and Kenneth H. Downing ${ }^{2}$

Keywords: Charging, resolution, transmission electron microscopy

Brief Title: Physical principles of charging

${ }^{1}$ Department of Molecular and Cell Biology, Stanley/Donner ASU, University of California, Berkeley, California 94720-3206

${ }^{2}$ Life Sciences Division, Lawrence Berkeley National Laboratory, University of California, Berkeley, California 94720 


\begin{abstract}
While the most familiar consequences of specimen charging in transmission electron microscopy can be eliminated by evaporating a thin conducting film (such as a carbon film) onto an insulating specimen, or by preparing samples directly on such a conducting film to begin with, a more subtle charging effect still remains. We argue here that specimen charging is in this case likely to produce a dipole sheet rather than a layer of positive charge at the surface of the specimen. A simple model of the factors that control the kinetics of specimen charging, and its neutralization, is discussed as a guide for experiments that attempt to minimize the amount of specimen charging. Believable estimates of the electrostatic forces and the electron optical disturbances that are likely to occur suggest that specimen bending and warping may have the biggest impact on degrading the image quality at high resolution. Electron optical effects are likely to be negligible except in the case of a specimen that is tilted to high angle. A model is proposed to explain how both the mechanical and electron-optical effects of forming a dipole layer would have much greater impact on the image resolution in a direction perpendicular to the tilt axis, a well-known effect in electron microscopy of twodimensional crystals.
\end{abstract}




\section{INTRODUCTION}

It is common knowledge that the most severe manifestations of specimen charging that occur in transmission electron microscopy can be eliminated by either preparing samples directly on thin carbon films or by evaporating a thin carbon film onto the specimen after preparation. Included among the charging effects that can be controlled in this way are severe distortions and improper focusing of spots in electron diffraction patterns; image astigmatism that varies with the sample position; conspicuous, beam-induced movement of irradiated areas of the sample; rupture of thin films; and strong contrast effects at sharp or broken edges of the specimen. Examples of specimens in which severe charging is observed include thin sections of plastic-embedded cells and tissue; polymer films (formvar, collodion etc.); and specimens consisting of selfsupported thin films of ice (Jakubowski et al., 1989) or glucose (Brink et al., 1998b), spanning open holes within a carbon film. While the charging effects observed on selfsupported, nonconducting specimens are greatly reduced when an objective aperture is in place, they often are eliminated altogether by use of quite thin carbon films, as mentioned above.

In light of the well-known effectiveness of carbon films in preventing the various symptoms of specimen charging described above, it was a surprise to learn that a more subtle manifestation of specimen charging still remains for samples that are prepared on (or with) carbon films - and often for bare carbon films themselves. This remaining form of specimen charging can be seen as a phase-contrast "footprint" that is left in the area of the specimen that was just previously illuminated by the electron beam (Brink et al., 1998b). The contrast of this footprint is normally very weak, and one must use a low 
image magnification and very large defocus to see it. The effect is easily missed, ignored, or mistaken as an artifact of the fluorescence lifetime of the viewing screen, since the charging-footprint saturates very rapidly when the sample is shifted to a new position or when the beam diameter is increased to illuminate a larger area than before. Although there is no extensive description in the literature of this remaining charging effect, it is well appreciated that Dr. John Berriman was the first person to report its occurrence in specimens of the type that are used in biological electron cryomicroscopy. Numerous research groups have since communicated amongst themselves their observations regarding the "Berriman effect".

The charging effect reported by Berriman results in visible image-contrast which reverses sign upon changing from underfocus to overfocus conditions. This contrast can be distinguished in four ways from the electron-beam footprints that are created by specimen-contamination: (1) the contrast of the specimen-charging footprint saturates: beyond a certain, relatively low exposure, no further increase in contrast occurs as the exposure is continued; (2) the limiting extent of contrast is reached after an exposure of only a few hundred electrons $/ \mathrm{nm}^{2}$, normally too small an exposure to lead to significant contamination; (3) the footprint is formed just as easily on samples cooled to liquid nitrogen temperature, even though the surface-diffusion required to cause beam-induced contamination can no longer occur; and (4) in most cases the contrast (or footprint) that is produced by illuminating one area is erased when a second, adjacent area is subsequently illuminated. The erasure of charging-induced contrast can be seen in the pattern produced during spot-scan illumination (Downing, 1991), as is shown in the 
accompanying paper (Downing et al., 2002), where only the last few spots in the raster retain the contrast produced by the Berriman effect.

The physical nature of specimen charging that occurs on samples prepared with carbon films clearly must be quite different from that which occurs on poorly conducting specimens. The electrical field strength within an insulating film that has been coated with conducting layers has been analyzed in the context of radiation damage and atomic/molecular desorption of anions (Cazaux, 1995), but the converse (a conducting film that is coated by insulating layers) remained outside the scope of that analysis. As there is no published discussion of what should be expected in the situation encountered during electron microscopy of biological macromolecules, and in order to provide background for the following paper that describes our own effort to mitigate the remaining charging effect, it has seemed appropriate to survey the physical principles that must apply to charging in such specimens. This survey is divided into three main sections. The first section argues that specimen charges should be organized as a dipole sheet (or opposed dipole sheets). The second section surveys the physical properties of the specimen and its environment that must determine the kinetics of charging and charge neutralization. The third section discusses the ways in which specimen charging - even in the presence of a conducting carbon film - is likely to limit the contrast of high resolution images recorded under low-dose conditions. This third section therefore serves to motivate the effort to reduce the amount of specimen charging that still remains on specimens prepared with the help of a conducting layer. 


\section{SPECIMEN CHARGING MUST BE COMPENSATED BY CAPACITATIVE CHARGING WITHIN THE CONDUCTING LAYER}

Simple physical reasoning requires that specimens in which a long-lived, beaminduced pattern of charging can be observed must contain a non-conducting layer, i.e. an insulating film, within which fixed charges can be built up. When evaporated carbon film is deposited onto a nonconducting sample, or conversely when a nonconducting sample is deposited onto a carbon-support film, the usual types of charging effects that are observed on self-supported insulating films (Jakubowski et al., 1989; Brink et al., 1998a) no longer occur. Contrary to earlier belief, however, the charges that otherwise build up in the insulating layer must not actually be neutralized by charges flowing from the conducting, carbon film (or at least not completely so), for then the charging-related image contrast reported by Berriman would not occur.

The simplest specimen structure that can produce the Berriman effect must therefore consist of an insulating layer and a conducting layer. Illumination of a defined area of such a specimen by the electron microscope beam will produce an area of fixed, positive charges within the insulating layer due to ionization and the resulting escape of secondary electrons. These positive charges must then be balanced, but not necessarily neutralized, by negative charges that can flow within the conducting layer.

To a first approximation, then, specimen changing that occurs in the presence of a conducting layer should resemble a dipole-sheet, like that which is formed in a parallel plate capacitor (Figure 1A). Thus, even though the fixed charges that are trapped in the insulating layer are not neutralized as such, the presence of a conducting layer (e.g., 
carbon film) ensures that there is no build up of net charge, unlike what happens in the case of a self-supported insulating film.

One might also expect to have situations in which there are non-conducting films on both surfaces of the conducting body of a carbon film, as in discussed below. In this case fixed, positive charges will be created on both sides of the conducting layer, as is illustrated in Figure 1B, although these need not be symmetrical in magnitude.

Non-conducting layers can occur on one or both sides of a carbon support film for a variety of reasons. (1) The biological specimen itself represents an insulating film. Thus, a polar structure of the type envisioned in Figure 1A would be created either by depositing the biological sample onto a support film or by evaporating carbon onto a selfsupported specimen. (2) The surface of a carbon film may itself have poor electrical conductivity due to intrinsic perturbations of its structure near to the surface or due to chemical reactions with the surrounding atmosphere, physical adsorption of gases, etc. An experimental measurement of the conductance of evaporated carbon films shows that the conductance increases linearly with thickness of the carbon film as it should do, but only after a given thickness of carbon has been deposited. In the example shown in Figure 2, the intercept is approximately $4 \mathrm{~nm}$, but this value varies for individual experiments. Since carbon films of half this thickness can be floated from a mica substrate and appear uniformly thick in the electron microscope, the poor conductivity at $20 \mathrm{~Hz}$ cannot be explained by a model in which islands of evaporated carbon have not yet become electrically connected to one another. (3) Ultimately one must work at low temperature with most biological specimens. This fact raises the possibility that thin films of vitreous ice will condense onto the surfaces of the conducting carbon film, 
thereby producing a more-or-less symmetrical sandwich of the type envisioned in Figure $1 \mathrm{~B}$.

Positive charges can be formed within the insulating film by either of two physical processes that differ from one another in a quite fundamental way. (1) The more obvious process simply involves the escape of secondary electrons that are produced by ionization. The measurement of escaping secondary electrons is, of course, one of the familiar ways in which image contrast is produced in the scanning electron microscope (Reimer, 1998; Joy, 1995). The resulting trapped, positive charges are located, for the most part, within the escape depth for secondary electron production, which may be as great as $5 \mathrm{~nm}$ or more. It is supposed that such trapped positive charges can be neutralized again by the capture of secondary electrons produced elsewhere, or by the capture of electrons excited into previously vacant conduction bands (electronic levels) of the insulating material. (2) In addition, however, we suggest that electron-donating sites, analogous to those on the positive side of a $\mathrm{p} / \mathrm{n}$ junction, might be created by radiolytic processes. The creation of such sites, which might be distributed homogeneously within the insulating film, must be postulated in order to account for a component of the specimen-charge contrast that is not erased when the electron beam is moved to a new area.

There is at present no way to measure the charge density (charge per unit area) within the insulating layer. The charge density could, in principle, reach a value even larger than that which would build up in a corresponding, self-supported insulating film, yet the observable effects could be greatly reduced by the global charge-neutralization achieved with the help of a conducting film. Alternatively, the close proximity of a 
conducting layer might result in true neutralization of all but a small fraction of the charge that could otherwise be held on a self-supported insulating layer. Some of the principles involved that ultimately limit the maximum charge density and that determine the kinetic approach to the steady state charge distribution are discussed in the next section. For the moment we note only that a believable estimate of the charge density can be calculated from the incident electron exposure that leads to saturation of the contrast effect $\left(\sim 100\right.$ electrons $\left./ \mathrm{nm}^{2}\right)$ and from reasonable estimates of the secondaryelectron emission coefficient of thin insulating materials. In the case of a very thin film the secondary emission coefficient may be as low as 0.1 per cent. Taking this as a minimum value, one can estimate that the number of trapped positive charges generated (i.e., the number of secondary electrons produced) by a "saturating exposure" of 100 electrons $/ \mathrm{nm}^{2}$ would be 0.1 charges $/ \mathrm{nm}^{2}$.

To a rough approximation, the charge distribution that is expected to occur will be a dipole sheet rather than a layer of surface charge as has already been noted in Figure 1. For clarity we emphasize that we mean here the actual charge distribution and not the mathematical construction of image charges that is commonly used for electrostic calculations at a dielectric interface. The electric potential of a finite patch of dipoles (with a dipole moment per unit area designated by the symbol $\tau$ ) is itself easily represented, in the region outside the specimen itself, by the simple equation (Panofsky and Phillips, 1962)

$$
\mathrm{V}(\mathbf{r})=\frac{\tau}{4 \pi \varepsilon_{\mathrm{o}}} \Omega(\mathbf{r})
$$


where $\varepsilon_{\mathrm{o}}$ is the permitivity of free space and $\Omega$ is the solid angle subtended by the dipole sheet as viewed from the point of observation, $\mathbf{r}$, where $V$ is to be measured. However, the requirement that the electric field must be zero within the electrical conductor means that some of the negative charges must be distributed outside the disk of positive (i.e. fixed) charges, in effect acting as a guard ring (Figure 3) that prevents the electric field from penetrating into the conducting layer. The small amount of uncompensated, positive charge that thus is postulated to remain within the illuminated area could provide the basis for the faint amount of phase contrast which is seen in the "Berriman effect."

\section{KINETIC FACTORS MUST CONTROL THE FORMATION, SATURATION AND NEUTRALIZATION BEHAVIOR OF THE BERRIMAN EFFECT}

Since there has not yet been a previously published discussion of the physical processes that account for the Berriman effect, we believe that it is appropriate to make a first attempt to identify at least the more obvious factors that need to be considered. Development of a physical model that describes the Berriman effect, even in a qualitative form, can serve as a conceptual framework for the design of experiments that might minimize this form of specimen charging.

We are inclined to think of the specimen charging that occurs in this situation in terms of an immobile surface-density of positive charges, $\sigma$, that must build up within an insulating layer on the surface of the specimen. More specifically, we expect the immobile charges to be confined within the "secondary-electron escape depth", relative to the solid-vacuum surface. Although we speak of a surface-charge density, we take it 
for granted that negative countercharges will flow in the underlying "conducting" layer, thereby setting up a dipole-layer density, $\tau$, rather than an uncompensated surfacecharge density, $\sigma$. This fact is assumed implicitly in what follows, and thus we discuss only the positive surface-charge component of $\tau$, which we refer to by the symbol " $\sigma$ ".

The initial rate of charging, i.e., $\frac{d \sigma}{d t}$, will necessarily be proportional to the incident beam intensity, $\mathrm{I}_{\mathrm{o}}$ (electrons/s-area). The surface-charge density must ultimately saturate, due to the progressive increase in the positive potential at the free surface. In addition, however, it is likely that there are other competing processes that result in neutralization or "discharging" of the positive-charge surface density. We believe that the simplest model for neutralization should include one term that depends upon the thencurrent value of $\sigma$ and a second term that depends on the difference between $\sigma$ and an offset value, $\sigma_{0}$. Thus, our starting model would be summarized by the differential equation;

$$
\frac{d \sigma}{d t}=\mathrm{k}_{1} \mathrm{I}_{\mathrm{o}}-\mathrm{k}_{2} \mathrm{f}_{2}(\sigma)-\mathrm{k}_{3} \mathrm{f}_{3}\left(\sigma-\sigma_{\mathrm{o}}\right)
$$

The physical processes that determine the rate constants $\mathrm{k}_{1}, \mathrm{k}_{2}$ and $\mathrm{k}_{3}$ will be discussed below. The mathematical form of the functions $f_{2}(\sigma)$ and $f_{3}\left(\sigma-\sigma_{0}\right)$ cannot be specified in advance, and therefore they represent no more than a lumped, conceptual representation of all processes that lead to neutralization of the positive surface-change, $\sigma$.

The rate constant for charge buildup, $\mathrm{k}_{1}$, should depend primarily on the secondary-electron emission coefficient. This rate constant may vary from one type of insulating material to another, and it may also depend upon the energy of the incident 
electrons. All things considered, however, we do not expect that $\mathrm{k}_{1}$ will show a major variation from one experimental situation to another.

The term $k_{2} f_{2}(\sigma)$ in equation 2 is meant to represent, symbolically, all process that decrease rather than increase the amount of positive surface charge, $\sigma$, and do so in a way that depends only on the value of $\sigma$. Examples of the processes that contribute to charge neutralization in this way would include (1) the flow of an Ohmic "leakage current" through the insulating material, (2) electron tunneling between the conducting layer and the region of immobile positive charge, if the insulating layer is very thin, (3) the capture of low-energy secondary electrons generated when scattered electrons hit the lens pole pieces, the objective aperture, etc., or even (4) the capture of electrons excited into empty conduction bands of the insulator by inelastic scattering processes in an adjacent area. In reality each such process would certainly occur with its own separate rate constant and even with its own form of non-linear dependence on $\sigma$.

The term $\mathrm{k}_{3} \mathrm{f}_{3}\left(\sigma-\sigma_{\mathrm{o}}\right)$ in equation 1 is meant to represent all processes that can neutralize the positive surface-charge, but can do so only above a threshold value of the surface charge. One example would be the formation of immobile charges in the insulating layer due to a radiation-induced change in the contact potential between the insulator and the conducting film. A second example of a term of this type, at least from an empirical point of view, would be neutralization that would occur when the voltage difference generated by a surface charge of $\sigma_{0}$ would exceed the breakdown strength of the insulating material.

A conceptually similar equation can be imagined that would describe the kinetics of neutralization of the surface charge, $\sigma$, built up in a previously irradiated area, once the 
beam is moved to an adjacent area of specimen. In this case, the only processes that are likely to be significant are neutralization (1) by low energy secondary electrons (produced by impact of primary electrons on the specimen and by scattered electrons when they hit the objective aperture), and (2) by electrons excited into the empty conduction bands of the insulator as a result of inelastic scattering events in the adjacent area.

\section{SPECIMEN CHARGING CAN DEGRADE HIGH-RESOLUTION IMAGE CONTRAST}

Specimen charging might reduce the contrast recorded in high-resolution images in two distinct ways. (1) The mechanical stress resulting from Coulomb-repulsion between immobile, positive charges must be translated into mechanical strain (physical movement) within the specimen. (2) Electrostatic charging can lead to unwanted electron-optical perturbations.

In both cases the image contrast will be affected only if the amount of specimen charging changes with time during the exposure. Charging should have no effect on image quality after the system has reached a stable, steady-state condition, since both the mechanical stress and the electron optical effects would then be invariant in time. Unfortunately the "time-constant" for specimen charging (expressed in units of electron

exposure, electrons $/ \mathrm{nm}^{2}$ ) is similar to that for causing severe radiation damage (in biological specimens) at high resolution. As a result one does not have the option to illuminate the sample long enough to reach a steady state before starting to record a highresolution image. 
It is instructive to calculate the force due to Coulombic repulsion between two point charges at a distance of $\sim 3.2 \mathrm{~nm}$, the closest approach (on average) expected from the estimated upper bound on the charge density, that was given above. Assuming that the dielectric constant is 3 , this force would be $\sim 8 \times 10^{-12}$ Newtons. The mechanical stress that is caused by charging may even be much less than this estimate would indicate, however, because the forces between positive charges in the non-conducting specimen are substantially balanced by the negative charges in the conducting (e.g., carbon) film. The amount of strain (expansion) of a stiff material such as evaporated carbon that would be produced by such a small force is certain to be too small to affect the quality of highresolution images. Unfortunately, however, this is a large enough force to cause significant bending of a thin film, the actual amount of bending depending upon the geometry with which the thin film is attached to the more rigid specimen-grid. As a result, dynamic bending and warping of the thin-foil sample can occur while recording an image, and this form of specimen motion remains a possible reason for charging-induced degradation of image quality.

Buckling and warping of the specimen is likely to have an even more severe effect on image resolution with highly tilted samples than it would have on untilted specimens. In this case, the additional increment in how badly the image is degraded will be greatest in the direction perpendicular to the tilt axis. A representative calculation that serves to illustrate this point assumes a local, one-degree tilt of a $100 \mathrm{~nm}$ diameter patch of the specimen. Regardless of the direction of the tilt within the plane of the specimen, one end of the patch will shift "vertically" (i.e., perpendicular to the plane of the specimen) by $\sim 17$ Angstroms. When the sample is untilted, this change in sample-height 
would go unnoticed since it is much smaller than the depth of field at even the highest expected resolution. When the sample itself is tilted to a high angle, however, the "vertical" movement just mentioned will automatically have a large horizontal component in the direction perpendicular to the axis of the sample-tilt. At a sample-tilt of only 30 degrees, for example, the horizontal shift of the image will already be half of the "vertical" shift.

While there is no reason at present to believe that electron optical effects resulting from specimen charging are important in untilted samples, it is easy to see that they could account for a significant amount of image-deflection when recording low-dose images at high tilt angles. As an example, one can discuss the simplified case of an "infinite" dipole sheet, for which the electrostatic potential undergoes a step-change as the electron beam crosses the interface. As pointed out by Dr. Dieter Typke, this step-change in potential is the electron-optical equivalent of the air-glass interface in light optics. The incident electron beam will thus be deflected in a direction perpendicular to the tilt axis of the specimen, in the same way as is described by Snell's Law for the corresponding situation in light optics.

A physically realistic model, illustrated in Figure 4, has been used to confirm that specimen charging can result in significant deflection in the direction perpendicular to the tilt axis. The electrostatic potential in this model is assumed to jump discontinuously across the dipole layer by $\sim 10$ volts, the value appropriate to a dipole sheet with 0.1 positive charges $/ \mathrm{nm}^{2}$, separated vertically by a distance of $5 \mathrm{~nm}$ from a similar layer of negative charges. The deflection angle for a $300 \mathrm{keV}$ electron that is decelerated by $10 \mathrm{eV}$ as it crosses this interface would be only $\sim 2 \times 10^{-3}$ degrees $\left(\sim 3.5 \times 10^{-5}\right.$ radians $)$ if the 
sample (i.e., the dipole sheet) is tilted by 60 degrees. This deflection would of course result in a negligible displacement of the image (perpendicular to the tilt axis) if it were exactly compensated by an equal and opposite dipole layer on the bottom side of the specimen, as is shown in Figure 4. In the other extreme, however, if the bottom side of the specimen remained uncharged, the transmitted electron would not accelerate back to $300 \mathrm{keV}$ until the solid angle subtended by the dipole-patch approached zero steradians, i.e., at several times the diameter of the irradiated area of the sample. In this case the image displacement might easily reach $0.5 \mathrm{~nm}$ for an illuminated patch-size of $1 \mu \mathrm{m}$, thereby causing a major loss of contrast at a resolution of even $1 \mathrm{~nm}$.

The "electron refraction" model emphasizes that the amount of image-movement perpendicular to the specimen-tilt axis may depend greatly on (1) how symmetrical, or not, the dipole-sheets are on both sides of the sample (the best situation being for them to be perfectly symmetrical) and (2) how large the dipole moment per unit area actually is, the value used in these calculations being just a rough estimate. The appealing feature of this model is that it offers explanations for why the image quality (perpendicular to the tilt axis) could occasionally be very good, even when it is poor in the majority of the areas of the specimen.

\section{CONCLUSION}

The build up of positive charge near the surface of an insulating film is capable of degrading the quality of high resolution electron microscope images, even when the positive charge is fully compensated by negative counter charges that flow within an underlying, conducting layer of a thin specimen. The most likely cause of the degradation 
of image quality appears to be mechanical bending and warping of the thin-foil specimen due to forces generated by Coulombic repulsion between adjacent, immobile positive charges that are trapped in the insulating layer at the surface of the specimen. Dynamic bending and warping of the specimen is likely to cause especially severe loss of image quality in the direction perpendicular to the tilt axis for highly tilted specimens, but it can already degrade the quality of images of untilted specimens to a very significant extent.

It is unlikely that the image quality of untilted specimens is degraded by electronoptical effects resulting from charging since the surface layer of positive charge is largely compensated by capacitative charging within the conducting layer. On the other hand, significant electron-optical image shifts are possible with highly tilted specimens if the dipole moment per unit area is not perfectly symmetrical on the top and bottom surfaces of a thin sample. Thus the effect of dipolar charging adds to the already mentioned effect of bending and warping of the specimen, providing a second factor that can degrade the image quality to a much greater extent in the direction perpendicular to the tilt axis of the specimen.

In view of the fact that these qualitative arguments are supported by believable estimates of their magnitude, we conclude that it is possible that high-resolution image quality might be improved quite significantly if the residual charging effect, noted originally by John Berriman, can be reduced or eliminated by some form of experimental mitigation. This conclusion serves as the motivation for experimental work (reported in our companion paper) on the phenomenon that specimen charging still occurs, even when samples are prepared on a carbon film or when self-supported insulating specimens are coated by an evaporated carbon film. 


\section{ACKNOWLEDGEMENTS}

This work has been supported in part by National Institutes of Health Grant GM51487 and by the Office of Health and Environmental Research, U.S. Department of Energy under Contract DE-AC03-76F00098. 


\section{REFERENCES}

Brink, J., Gross, H., Tittmann, P., Sherman, M.B. and Chiu, W. (1998a) Reduction of charging in protein electron cryomicroscopy. J Microsc, 191, 67-73.

Brink, J., Sherman, M.B., Berriman, J. and Chiu, W. (1998b) Evaluation of charging on macromolecules in electron cryomicroscopy. Ultramicroscopy, 72, 41-52.

Cazaux, J. (1995) Correlations between ionization radiation damage and charging effects in transmission electron microscopy. Ultramicroscopy, 60, 411-425.

Downing, K. (1991) Spot-scan imaging in transmission electron microscopy. Science, 251, 53-59.

Downing, K.H., McCartney, M.R. and Glaeser, R.M. (2002) Experimental characterization and mitigation of specimen charging on thin films with one conducting layer. Submitted.

Jakubowski, U., Baumeister, W. and Glaeser, R. (1989) Evaporated carbon stabilizes thin, frozen-hydrated specimens. Ultramicroscopy, 31, 351-356.

Joy, D. (1995) Monte Carlo modeling for electron microscopy and microanalysis. Oxford University Press, New York.

Panofsky, W. and Phillips, M. (1962) Classical electricity and magnetism. AddisonWesley Pub Co, Reading, MA.

Reimer, L. (1998) Scanning electron microscopy: physics of image formation and microanalysis. Springer, Berlin; New York. 


\section{FIGURE LEGENDS}

Figure 1. Schematic diagram illustrating the distribution of fixed (positive) and mobile (negative) charges expected to form within a thin-film specimen after a brief period of illumination by the incident electron beam. Positive charges are expected to be randomly distributed in a thin layer near to the surface of an insulating layer, while negative charges will accumulate in the underlying conducting layer, distributed within the area opposite to the fixed, positive charges. (A) A cartoon-representation showing the positive charges confined to a depth corresponding to the secondary-electron escape depth, within an area corresponding to the "footprint" of the illuminating beam. If the thin-film specimen has an insulating layer on only one side, the charge distribution will be characterized by a given dipole movement per unit area, $\tau$. (B) If the thin-film specimen has insulating layers on both surfaces, there will be two dipole sheets that face in opposite directions.

Figure 2. Experimental measurement of the conductance of an evaporated carbon film as a function of film thickness (expressed in terms of the frequency shift of a quartz thickness-monitor). Two gold electrodes were initially evaporated onto a substrate. After electrical leads were attached to the gold, carbon was then evaporated onto the substrate. The conductance remained essentially zero until the thickness of evaporated carbon, as reported by the quartz monitor, reached approximately $4 \mathrm{~nm}$, after which the conductance increased linearly (with some deviations) with the thickness of evaporated carbon. The geometry of the sample and the thickness monitor, in relation to the carbon source, was such that $10 \mathrm{~Hz}$ corresponds to about $1 \mathrm{~nm}$ of carbon. 
Figure 3. Proposed model for the formation of a small amount of "uncompensated" positive charge within the footprint of the incident electron beam. (A) The fringing field at the edge of a dipole layer would produce an electric field within the conducting layer if the "footprint" of negative charge corresponded exactly to that of the positive charge. The charge distribution shown here would not be stable, of course, since charges would then flow within the conductor until the field strength there became zero. (B) If some of the negative charge extends beyond the "footprint" of the positive charge, the electric field within the conductor can be eliminated. The consequence, then, is that there is a residual amount of positive charge within the footprint of the incident electron beam that is not fully compensated by the underlying negative charges within the same area.

Figure 4. Schematic diagram of the refraction of an incident electron beam by a thin-film specimen within which the electrostatic potential jumps abruptly at the surface (e.g., due to the formation of a given dipole moment per unit area, $\tau$ ). The beam deflection will be perpendicular to the tilt axis of the specimen, and the amount of deflection will increase during irradiation, as the dipolar specimen-charging increases, until a saturating amount of charge has been established. The cartoon shows that the amount of deflection will be small if there are symmetrical dipole layers on both surfaces, but that the amount of deflection can be considerably larger if the value of the surface charge (the values of $\tau$ ) are not equal on the two surfaces, because the deflected beam is not immediately returned to its incident direction as it departs the bottom of the specimen. 

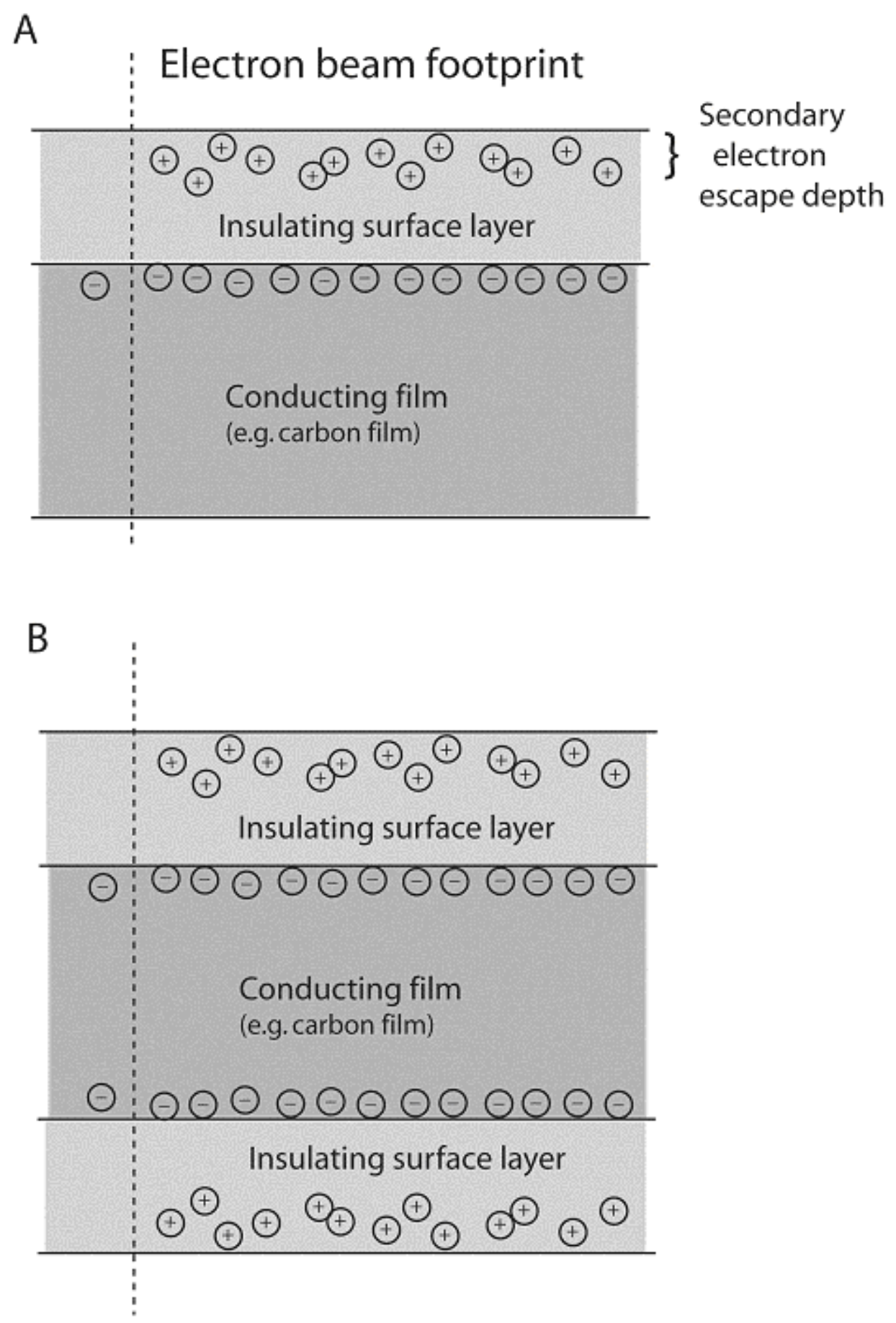

FIGURE 1 


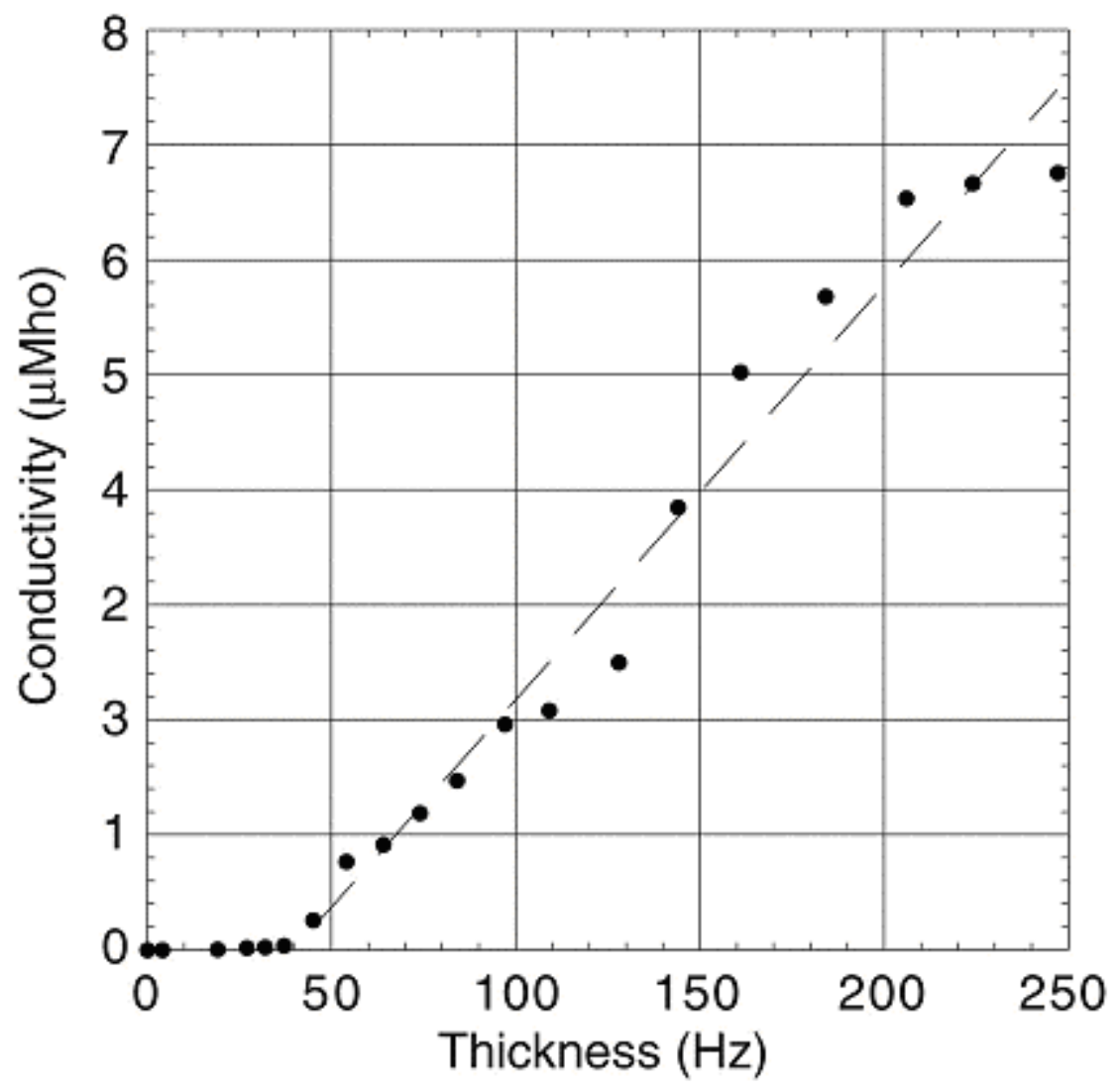

FIGURE 2 


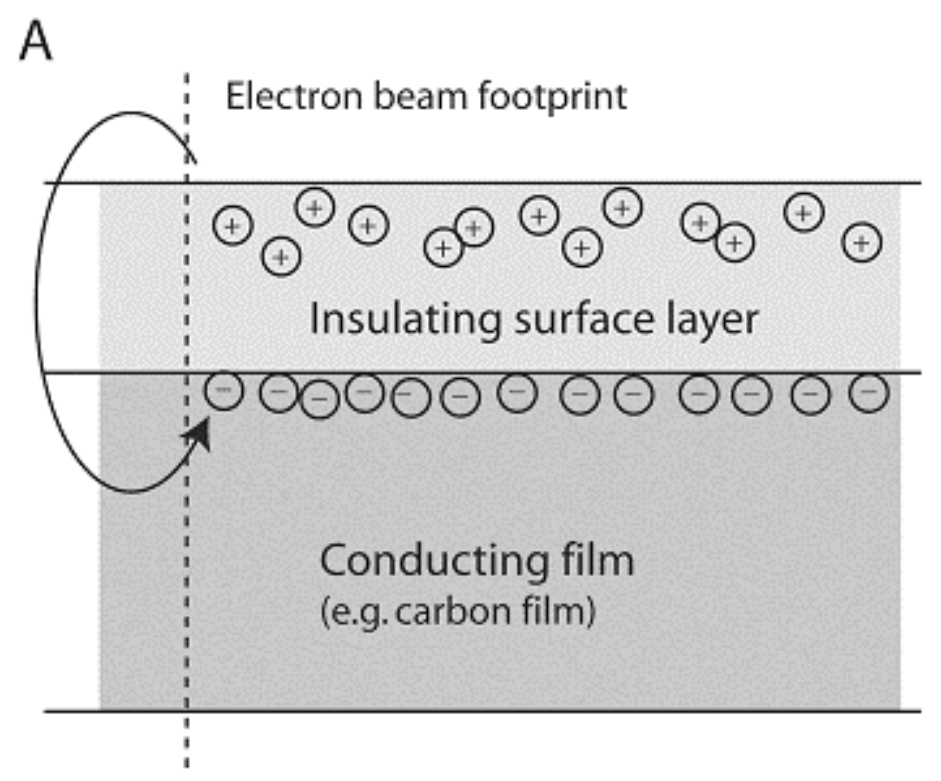

B

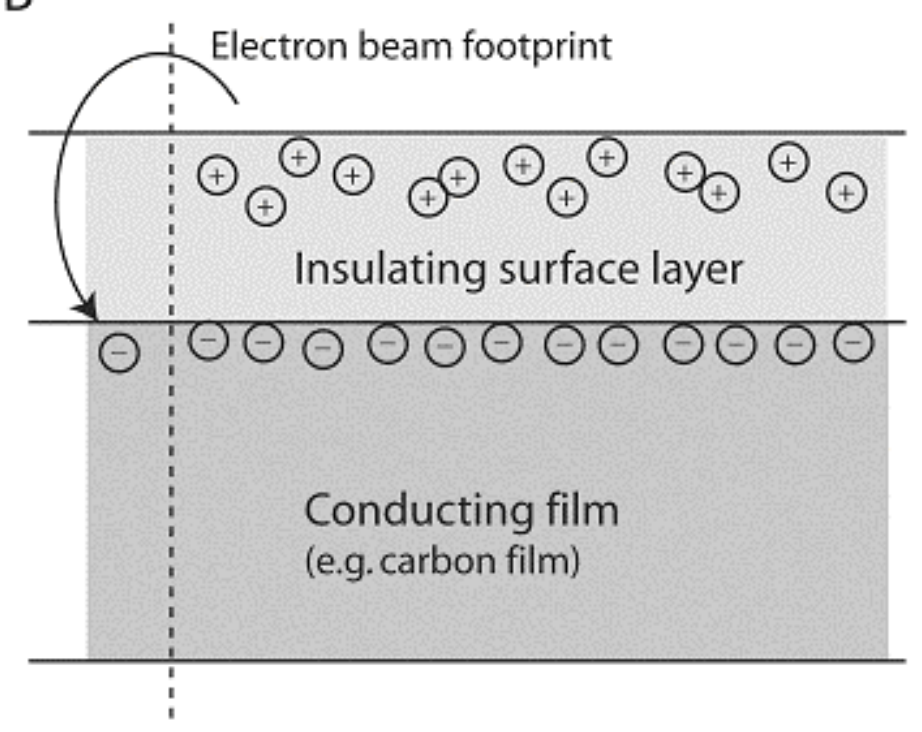

FIGURE 3 


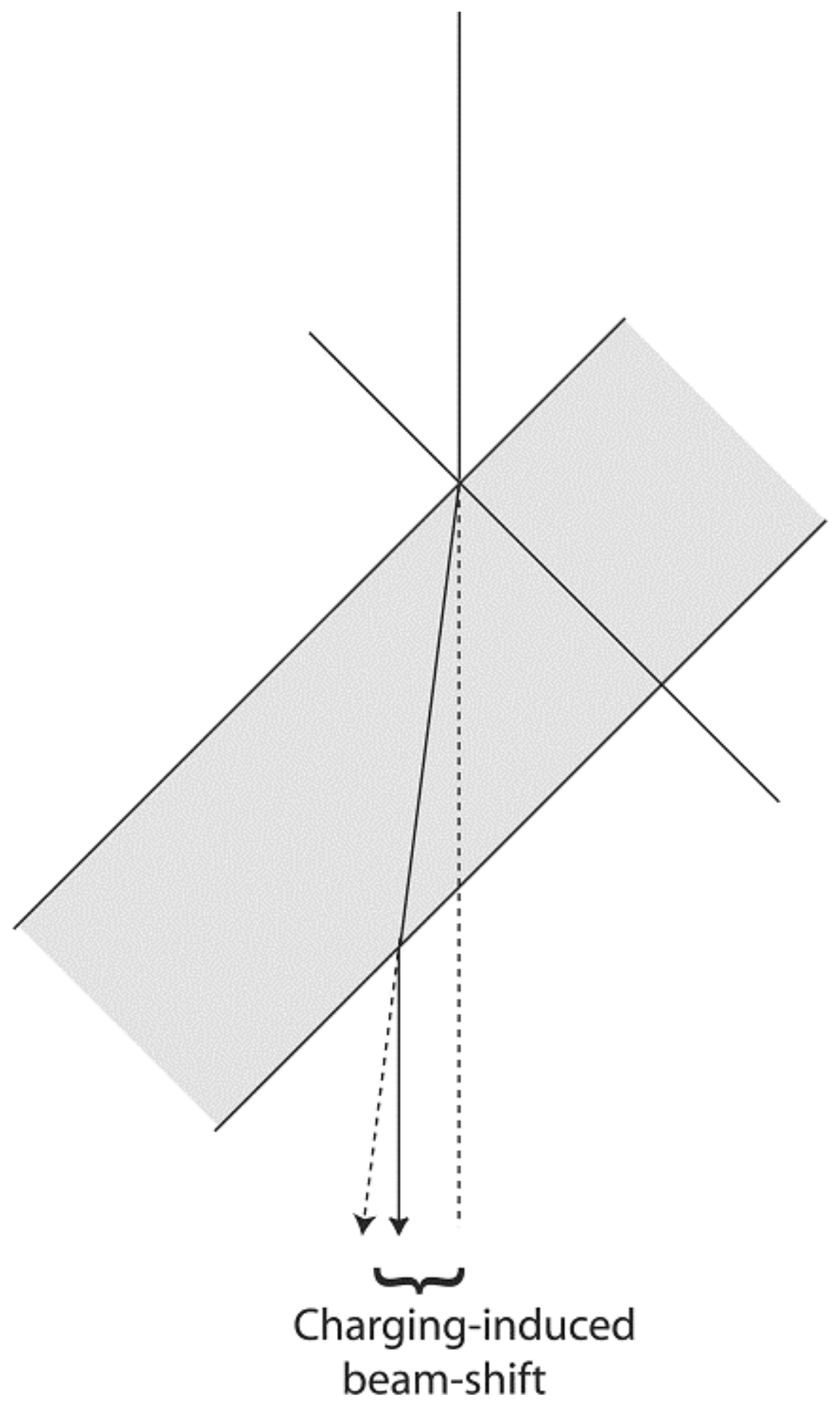

